\title{
Cyclotron spectroscopy of HU Aquarii ${ }^{\star}$
}

\author{
A. D. Schwope ${ }^{\star \star, 1}$, H.-C. Thomas ${ }^{2}$, K.-H. Mantel ${ }^{\star \star, 3}$, R. Haefner ${ }^{3}$, and A. Staude ${ }^{1}$ \\ 1 Astrophysikalisches Institut Potsdam, An der Sternwarte 16, 14482 Potsdam, Germany \\ 2 Max-Planck Institut für Astrophysik, Karl-Schwarzschild-Str. 1, 85740 Garching, Germany \\ 3 Universitäts-Sternwarte München, Scheinerstr. 1, 81679 München, Germany
}

Received 30 October 2002 / Accepted 13 February 2003

\begin{abstract}
We present the results of a combined analysis of low-resolution spectroscopy and high-speed optical photometry of the bright eclipsing polar HU Aqr, obtained in its high accretion state. Cyclotron harmonic emission in a field of $B \simeq 34 \mathrm{MG}$ became evident and could be traced through the whole optical bright phase. The cyclotron harmonics display an asymmetric motion as a function of phase which requires a certain forward tilt of the magnetic field lines in the emission region. The optical bright phase is more extended than the bright phase in soft X-rays suggesting a more extended emission region. Also, ingress into and egress from the optical eclipse last much longer in the optical than at X-ray wavelengths. These observations require the existence of an accretion arc with total extent of about $20^{\circ}$. The soft X-ray accretion spot is located at the far end of the arc, where the bulk of matter is accreted. The arc is likely to be more extended than the foot-line of field lines connecting to the ballistic accretion stream in a dipolar geometry. This together with the required tilt of the accreting field lines suggest a more complex magnetic geometry than a simple dipole.
\end{abstract}

Key words. accretion, accretion disks- stars: binaries: eclipsing - magnetic fields - stars: individual: HU Aqr

\section{Introduction}

HU Aqr is the brightest eclipsing polar (magnetic cataclysmic binary of AM Herculis type), both at optical and X-ray wavelengths. It was detected independently in the ROSAT-XRT and ROSAT-WFC all-sky surveys (Hakala et al. 1993; Schwope et al. 1993), where its binary nature and the eclipse were discovered. The eclipse length of 0.0783 phase units is the longest among all polars (in relative, i.e. phase units), the system has an inclination of the binary orbit of $85.6^{\circ}$.

The brightness of the system, $V_{\max } \simeq 14^{\mathrm{m}}$. 5 , ROSAT PSPC count rate up to $40 \mathrm{~s}^{-1}$, made detailed observational studies possible. The extent of the accretion stream was uncovered in Doppler maps based on high-resolution optical spectroscopy (Schwope et al. 1997). The brightness distribution along the accretion stream was addressed by different teams in several papers making use of high-resolution (spectro-) photometric observations around eclipse phase in low and high accretion

Send offprint requests to: A. D. Schwope,

e-mail: aschwope@aip.de

* Based on observations at the European Southern Observatory, Chile, at the ESO $1 \mathrm{~m}$ telescope and the ESO/MPG $2.2 \mathrm{~m}$ telescope in Max-Planck-time.

$\star \star$ Visiting astronomer, German-Spanish Astronomical Center, Calar Alto, operated by the Max-Planck-Institut für Astronomie, Heidelberg, jointly with the Spanish National Commission for Astronomy. states (Hakala 1995; Harrop-Allin et al. 1999, 2001; Vrielmann \& Schwope 2001).

An extended monitoring campaign with the ROSAT and EUVE satellites allowed the study of the coupling of matter onto magnetic field lines and its dependence on the accretion rate. In addition, detailed modelling of the X-ray emitting hot spot in the high accretion state was possible (Schwope et al. 2001). The soft X-ray emitting spot was found to have a latitudinal extent of about $450 \mathrm{~km}\left(3^{\circ}\right.$ full opening angle). It was also vertically extended by $0.015 R_{\mathrm{wd}}$ (white dwarf radii) with respect to the undisturbed white dwarf photosphere. An accretion curtain of matter that has left the ballistic accretion stream was inferred based on an X-ray absorption map. The matter at the corresponding accretion arc (foot-line of accreting field lines) was not found to emit X-rays. Soft X-rays seem to be originating mainly from the densest part of the accretion stream at the end of the accretion arc.

The magnetic field strength of HU Aqr was determined by Schwope et al. (1993) as $B \sim 37 \mathrm{MG}$, and by Glenn et al. (1994) as $B=36 \pm 1 \mathrm{MG}$. Both determinations were based on the identification of cyclotron harmonics in low-resolution spectra obtained in low or intermediate accretion states.

Here we present the results of low-resolution spectroscopy with full phase coverage obtained in intermediate and high accretion states. We study the phase-dependent motion of individual cyclotron harmonics, decompose the optical $R$-band light curve and separate the cyclotron component. Both, the motion of the cyclotron humps and the light curve of the cyclotron 
Table 1. Optical obervations of HU Aqr discussed in the present work.

\begin{tabular}{lllcc}
\hline \hline Date & Telescope & Instrument & $\begin{array}{c}\text { No spectra/ } \\
\text { hours photometry }\end{array}$ & time resolution \\
\hline $1992 / 09 / 28$ & ESO150 & B\&C & 20 & $390 \mathrm{~s}$ \\
$1993 / 08 / 18-19$ & ESO/MPG220 & EFOSC2 & 40 & $6 \mathrm{~m}$ \\
$1993 / 08 / 17,18,21$ & ESO100 & $B$-band & $4.4,2.9,4.6 \mathrm{~h}(7 \mathrm{ecl})$ & $2 \mathrm{~s}$ \\
$1993 / 08 / 16-18$ & CA220 & MCCP $(U B V R I)$ & $5.7,6.8,3.5 \mathrm{~h}(9 \mathrm{ecl})$ & $0.5 \mathrm{~s}$ \\
\hline
\end{tabular}

component are clearly different from expectations based on the $\mathrm{X}$-ray geometry suggesting an origin of the optical cyclotron and the soft X-ray radiation at different places. We propose a magnetic geometry that implies non-dipolar field components and determine the likely extent of the accretion arc in HU Aqr, which fits the observed motion of cyclotron humps and detailed optical eclipse light curves.

\section{Observations}

HU Aqr was observed with the ESO 1.5m telescope for three hours on September 28, 1992, between UT 2:10 and 5:10. The telescope was equipped with the Boller \& Chivens spectrograph. A total of 20 low-resolution spectra with integration times $390 \mathrm{~s}$ were taken under good photometric conditions. Arc-lamp and flat-field spectra were taken regularly, the spectrophotometric standard star Feige 110 was observed immediately after HU Aqr. The spectra with a resolution of $\sim 8 \AA$ ( $F W H M$ of arc lines) cover the whole optical wavelength range 3300-10000 A.

The binary system was re-observed with the ESO/MPG $2.2 \mathrm{~m}$ telescope on August 18 and 19, 1993, between 00:303:00, and 1:00-3:15 UT, respectively, i.e. full phase coverage of the $125 \mathrm{~min}$ binary was achieved during all nights of observations. The instrument used was the ESO Faint Object Spectrograph and Camera (EFOSC2) with the G1 lowresolution grism. This combination gives low-resolution spectra $(F W H M \sim 40 \AA)$ covering the wavelength interval between 3500 and $9000 \AA$. In each night 20 spectra were taken with integration times of 6 and $5 \mathrm{~min}$, respectively. The layout of the observations was similar as above with frequent arc-lamp, flat-field and standard star observations. The estimated photometric accuracy of our observations is of the order of $30 \%$.

The 1993 observations formed part of a multi-telescope campaign of quasi-simultaneous observations of HU Aqr using four different telescopes at Calar Alto, Spain, and La Silla, Chile. At La Silla we were using the $1 \mathrm{~m}$ photometric telescope for high-speed (2s) photometric observations in the $B$-band and, as described above, the 2.2-m telescpe with EFOSC2. At Calar Alto high-time resolution observations were performed at the $2.2 \mathrm{~m}$ telescope with the MCCP and the double-beam TWIN spectrograph. The MCCP (Barwig et al. 1987) allows simultaneous high-speed photometry of a target star, a comparison star and the night sky in five spectral bands ( $U B V R I)$. These data, taken with a time resolution of $0.5 \mathrm{~s}$, were used in order to calibrate photometrically the high-resolution spectra recorded with the TWIN (Schwope et al. 1997) and the lowresolution spectra presented here. The $1 \mathrm{~m}$ telescope was used as a kind of backup telescope, the data obtained with this telescope were found to be almost identical to the MCCP data with two small exceptions. The observations at La Silla in each of these nights were performed in binary cycle plus one compared to the Calar Alto observations. The detailed comparison of the two $B$-band light curves shows, that they are shifted by $1 \mathrm{~s}$ with respect to each other. The origin of this shift remained unclear and this limits the accuracy of our analysis. Fortunately, the $1 \mathrm{~s}$ uncertainty is not critical for the analysis. For the analysis in this paper we make use of the $B$ - and $R$-band data only, the $R$-band nicely traces one of the cyclotron harmonics, the $B$-band gives an idea about the additional radiation components in the optical wavelength range. Essential in the context of this paper is only the $R$-band light curve. Photometric calibration of our low-resolution spectroscopy was performed by folding the spectra through the spectral bandpasses of the MCCP and comparing the corresponding brightness values with the MCCP light curves. For that exercise we were using phase-averaged optical light curves.

The phase convention used throughout this paper refers to superior conjunction of the white dwarf (Schwope et al. 2001)

$\operatorname{BJED}\left(T_{0}\right)=2449217.345872(35)$

$$
+E \times 0.086820416195(47)
$$

(BJED: barycentric Julian ephemeris day).

\section{Light curves, spectra, and cyclotron humps}

HU Aqr has a self-eclipsing geometry of the accretion spot, i.e. the spot rotates behind the limb of the white dwarf for a certain phase interval. The optical light curves are therefore modulated by the spot geometry, the phase-dependent beaming of the cyclotron radiation, accretion-rate variations, eclipses by the secondary and the stream, and contributions from the secondary. In September 1992 the binary was encountered in an intermediate accretion state with orbital variability outside eclipse between $\sim 16.5$ mag in the faint phase and $\sim 15.8$ in the bright phase. In August 1993 HU Aqr was in a high accretion state with orbital variability (again outside eclipse) between $V=14^{\mathrm{m}} \cdot 6-15^{\mathrm{m}} \cdot 1$.

Representative bright- and faint-phase spectra obtained in the 1993 high state are reproduced in Fig. 1. The intermediate state spectra are very similar to those shown in the diagram and need not to be reproduced separately. They show the hallmarks of AM Herculis stars (polars), broad and asymmetric emission lines of hydrogen and helium, a strong Balmer jump in emission, and cyclotron harmonic emission lines in the bright phase, which are, in the case of HU Aqr, visible even in the unprocessed spectra. The numbers given in the lower panel of Fig. 1 


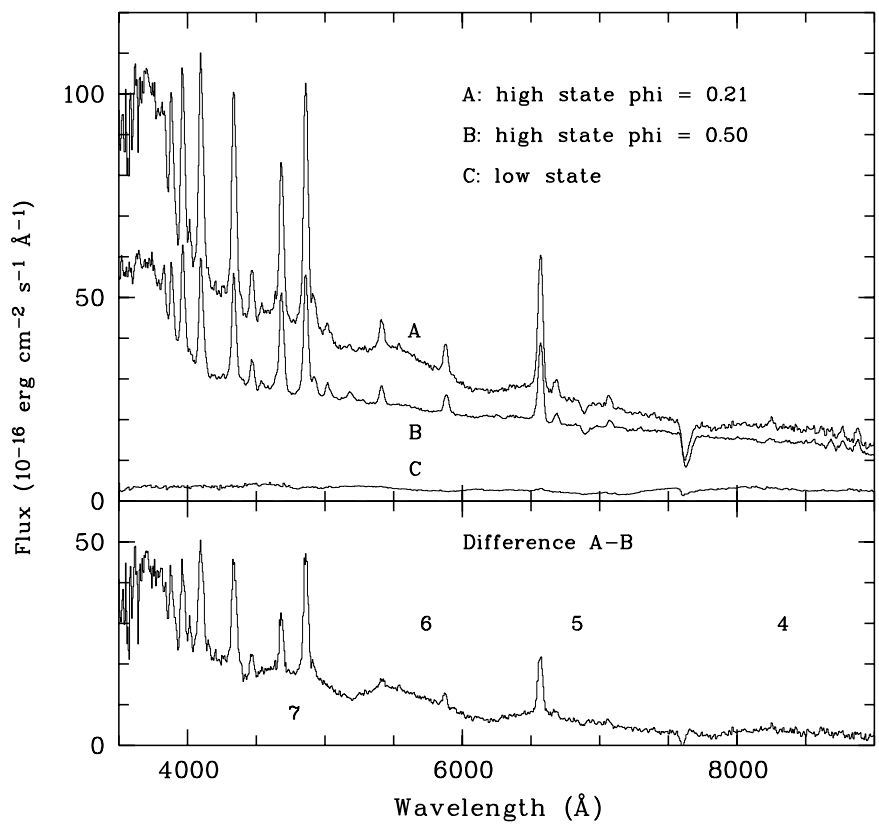

Fig. 1. Low-resolution spectra of HU Aqr obtained August 1993 with EFOSC2 at the 2.2-m telescope. In the upper panel the spectra " $A$ " and " $B$ " at given phases represent typical bright- and faint-phase spectra in the high-accretion state, respectively. The spectrum labeled " $C$ " indicates our low-state discovery spectrum obtained in August 1992 with the same instrument (Schwope et al. 1993). In the lower panel the difference $A-B$ is shown, which, to a good approximation may be regarded as pure cyclotron spectrum at phase $\phi=0.21$. The numbers indicate individual cyclotron harmonics. Binary phase 0.0 indicates superior conjunction of the accreting white dwarf.

indicate the integer harmonics of the cyclotron fundamental $\lambda_{1}=2 \pi c^{2} m_{e} /(e B)$, which lies, according to the field strength of about $34 \mathrm{MG}$, in the infra-red spectral range at $\sim 3 \mu$.

Cyclotron emission in an AM Herculis star originates from the heated plasma in the accretion regions, which are typically offset by some $15^{\circ}$ from the magnetic poles. If shock heating is important, one may reasonably expect a close vicinity of the cooling X-ray and optical emission regions and some similarity of the light curves (at least in geometrical terms, not in the angular characteristics). We therefore compare in Fig. 2 soft $\mathrm{X}$-ray and optical light curves. The soft X-ray light curve is based on a ROSAT-observation performed between Oct. 27 and Nov. 5, 1993, i.e. about 70 days after the optical observations, also in a high accretion state. The soft X-ray light curve is discussed in detail in Schwope et al. (2001), however, we describe the main features here briefly.

The soft X-ray light curve is modulated by $100 \%$ due to the stellar eclipse (at phase $\phi=0$ ), the self-eclipse of the accretion spot (in the phase interval $\sim 0.25-0.5$ ), and a preeclipse dip centred on phase $\sim 0.88$. The latter dip is due to absorption of X-rays in the accretion stream, which has just left the orbital plane. The phase of this feature indicates the azimuth $\chi_{\text {coup }}$ of the coupling region, where the bulk of matter couples onto magnetic field lines, $\chi$ coup $=43^{\circ}$. In a coordinate system fixed in the white dwarf the azimuth or longitude is measured with respect to the line joining both stars in mathematical positive direction. The centre of the bright phase allows

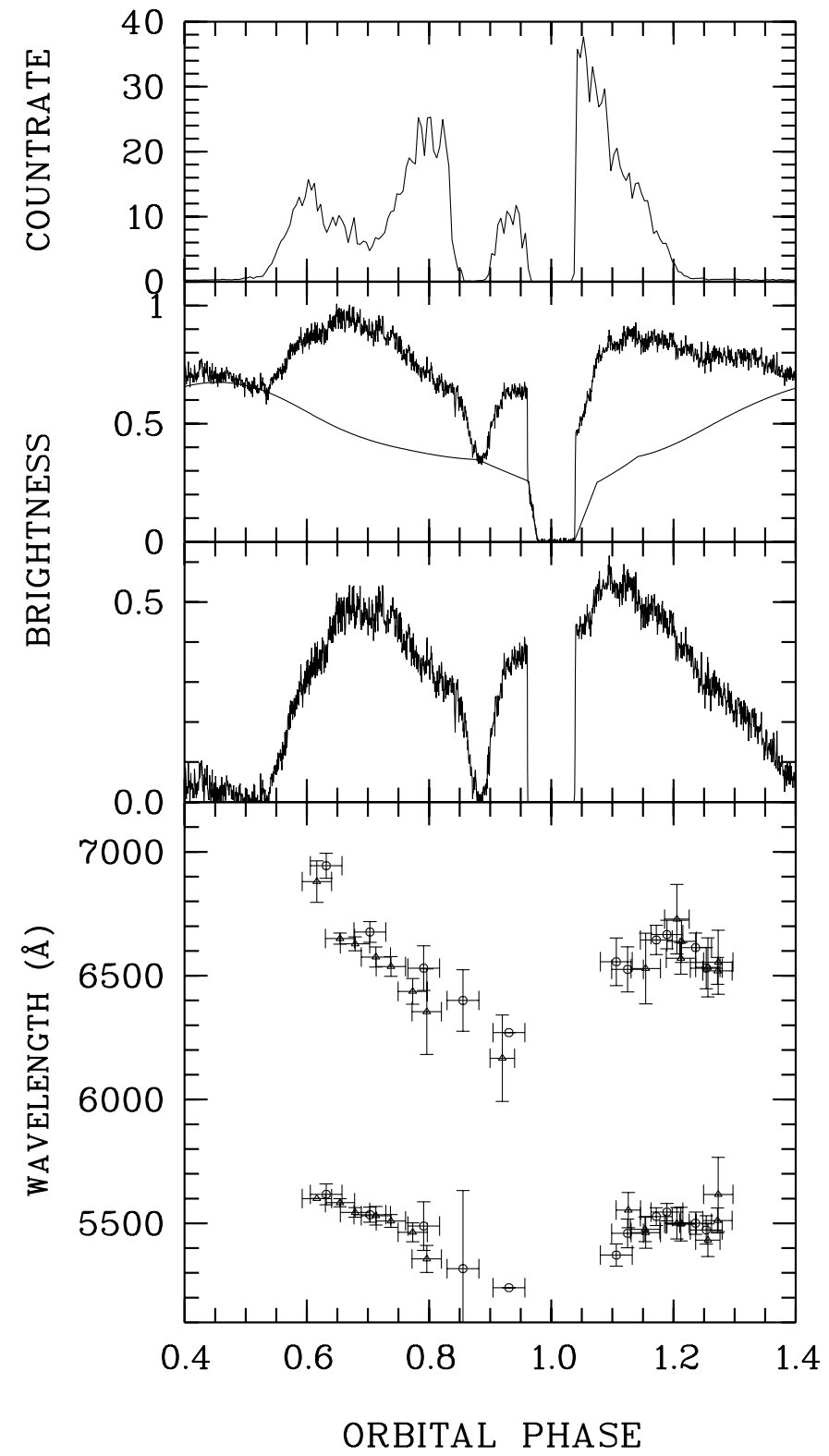

Fig. 2. (From top to bottom) (top panel) ROSAT-PSPC light curve obtained in October/November 1993 in a high accretion state. (second panel) Optical $R$-band photometry obtained with the MCCP at Calar Alto quasi-simultaneously with the low-resolution spectra shown in Fig. 1. The MCCP $R$-filter is effectively transmitting between 5700 and $6900 \AA$. The smooth curve below the observed light curve represents non-cyclotron components (see text for details). Units are arbitrary, i.e. plotted are relative count rates with respect to a nearby comparison star. (third panel) Difference of the two light curves in the second panel. This curve is regarded as pure cyclotron radiation in the 5 th harmonic. (bottom panel) The position (wavelength) of the 5 th (at $6500 \AA$ ) and 6th harmonics (at $5500 \AA$ ) as a function of binary phase.

to determine the azimuth of the accretion spot on the white dwarf surface, $\chi_{\text {spot }}=46^{\circ}$. Once the azimuth of the spot is fixed, the offset of the eclipse of the accretion spot with respect to true phase zero, about $7 \mathrm{~s}$, determines the co-latitude of the spot, $\delta_{\text {spot }} \simeq 30^{\circ}$ (measured with respect to the rotation axis). The time interval of eclipse ingress and egress determines the latitudinal extent of the spot, $\Delta \chi_{\text {spot }}=3^{\circ}$ (full opening angle). 
Once $\chi_{\text {spot }}, \delta_{\text {spot }}$, the latitudinal extent, and the orbital inclination are fixed, $i=85.6^{\circ}$, the length of the bright phase determines the vertical extent of the soft X-ray emission region, which is $h_{\text {spot }} \leq 0.015 R_{\mathrm{wd}}$. The temperature in the soft X-ray emitting spot was found to be $250000 \mathrm{~K}\left(k T_{\mathrm{bb}}=22 \mathrm{eV}\right)$.

The optical light curve shown in the second panel of Fig. 2 is the MCCP $R$-band light curve obtained quasi-simultaneously with the spectroscopy shown in Fig. 1 at the Calar Alto observatory. We investigate this light curve here in more detail (instead of the $U B V$ - or $I$-band data), since it is much less affected by optical line emission and photospheric emission from the white dwarf than the data obtained at shorter wavelengths, and has a much higher signal-to-noise ratio than the $I$-band data (due to higher throughput of the instrument and the higher number of photons offered by the source). The $R$-band effectively operates between 5700 and $6900 \AA$, i.e. it fully covers the 5 th cyclotron harmonic (Fig. 1). There is only one strong emission line in the $R$-band, namely $\mathrm{H} \alpha$. We determined the contribution of this line to the total signal in the $R$-photometry by folding the low-resolution spectra through the transmission curve of the $R$-filter, both before and after subtraction of the $\mathrm{H} \alpha$ line. It was found to contribute dependent on phase, reaching a maximum of $4 \%$ at phase 0.3 and remaining constant between $\phi=0.5-0.9$ at a $2 \%$ level. Hence, the contribution of optically thick emission lines to the $R$-band photometry is negligible at all phases (for emission line light curves of HU Aqr see Schwope et al. 1997).

The optical light curve has a maximum at phase 0.65 , it shows the pre-eclipse dip at phase 0.88 , a structured eclipse, a secondary maximum at phase 0.15 , and a faint phase between $\phi=0.35-0.53$. The optical pre-eclipse dip is centred on the same phase as in the X-ray light curve. Since the phase of this feature is an indicator of the accretion rate (Schwope et al. 2001), at higher rate the matter couples further down the stream, we may reasonably regard the X-ray light curve as representative for the optical observations performed two months earlier.

We separated the cyclotron contribution to the $R$-band light curve based on the following assumptions:

- the contribution of optical emission lines is negligible, i.e. below 5\%;

- the recombination continuum from the accretion stream is optically thin, i.e. constant throughout the orbital cycle (apart from the eclipse);

- in the centre of the optical pre-eclipse dip the cyclotron component is absorbed completely, i.e. all residual emission in the dip is recombination radiation from the stream ( 0.34 units in Fig. 2, second panel);

- the excess faint phase emission is fed by recombination radiation originating from the secondary star. Schwope et al. (1997) derived the corresponding light curve; a scaled version is added to the recombination component from the stream;

- the initial steep decline at eclipse egress is due to the obscured white dwarf. The remaining emission during the eclipse is recombination radiation from the stream. The slow decrease between dip and eclipse ingress is approximated linearly. A similar two-step function representing the contribution of the stream between $\phi=0.00-0.14$ is subtracted at egress phase.
Phase 0.14 is the latest possible egress phase according to the cone of the secondary's Roche lobe.

The resulting curve representing all non-cyclotron components is also plotted in the second panel of Fig. 2. The residuum after subtraction of the two curves is shown in the third panel of Fig. 2. We regard this as the cyclotron contribution to the $R$-band, emitted mainly in the 5 th cyclotron harmonic. The resulting light curve is double-humped due to cyclotron beaming with a minimum at phase $\sim 0.85$. The optical bright phase starts simultaneously with the X-ray light curve but lasts significantly longer (up to phase 0.35-0.40). The steps at ingress and egress of the white dwarf are unequal, an effect of the beaming characteristics of the cyclotron radiation. This result is not dependent on the details of the deconvolution procedure, since the steps can be directly measured in the observed light curves.

We measured the positions of cyclotron harmonic maxima following the procedure described by Cropper et al. (1989). The emission lines were approximated by single or double Gaussians and subtracted. The overall trend of the spectra was described by a low-order polynomial and also subtracted. The positions (wavelengths) of cyclotron harmonic peaks in the remaining normalized spectra were determined by fitting Gaussian curves. In the fourth panel of Fig. 2 we show the results of this procedure for all our low-resolution spectra obtained in 1992 and 1993 which displayed cyclotron harmonic emission (only fifth and sixth harmonics are shown). The vertical error bars represent the statistical error of the fitting process, the horizontal error bar the phase coverage of the individual spectrum. There is a clear, asymmetric motion of the wavelengths of the cyclotron humps by several hundred Angstrøms. The humps start at maximum redshift at the start of the bright phase and then move monotonically as a function of orbital phase towards shorter wavelengths. In the centre of the bright phase the observation of cyclotron humps is hampered by the absorption dip and the stellar eclipse. At later phases, i.e. after the eclipse, the harmonics move longwards until a maximum is reached shortly before $\phi=0.2$ and then again they are blueshifted. Cyclotron harmonic humps can be recognized in the spectra up to phase 0.3 , i.e. much longer than the $\mathrm{X}$ ray bright phase lasts. The 1992 and 1993 observations yield a consistent picture, although taken at different brightness level (accretion rate).

\section{Models for the cyclotron emitting region in HU Aqr}

Cyclotron harmonic motion was recognized earlier in V834 Cen (Schwope \& Beuermann 1990), UZ For (Schwope et al. 1990), MR Ser (Wickramasinghe et al. 1991), and in VV Pup (Schwope \& Beuermann 1997). In all these cases the motion of cyclotron harmonic humps was explained as being the result of a different aspect angle of the accreting field line in the hot accretion spot while the white dwarf rotates. Since, at the typical plasma temperature of a few $\mathrm{keV}$, the wavelength of a cyclotron harmonic depends on the angle between the magnetic field and the observers direction, the information contained in the motion of the humps can be used to infer the accretion geometry (or at least to check for consistency with other 
methods like polarimetry). There are more parameters which influence the position of a cyclotron harmonic: the magnetic field strength $B$ and the plasma temperature $T$ (for a detailed comparison of these effects, see Schwope 1990). As long as the accretion spot may be regarded as a small structure, wavelength shifts due to $B$ - or $T$-variations are negligible, since the observer always sees the same plasma. The situation may change, if extended accretion structures like accretion arcs are taken into account.

\subsection{Cyclotron emission from an accretion spot}

We start our modeling assuming the simple geometry of a point-like accretion region. The term "point-like" means and implies, that likely inhomogeneities in the temperature, the magnetic field strength, the plasma density, the field vector, the height of the emission region can all be ignored, since their effects are small compared to the beaming effects. We attempt to fit with such a model the cyclotron light curve (in particular its length and phasing and the double-hump structure), and the phase-dependent motion of the cyclotron humps.

In this scenario the motion of the humps is explained by a certain tilt of the local field vector in the accretion spot. The reversal at $\phi \simeq 0.2$ indicates the phase where the local field in the accretion spot is perpendicular to the observer. At later phases the accreting field line is seen from "below", a reversal of the sign of circular polarization is expected (Stockman 1988). In Stockman's terms the observer is below the polarization horizon. Sohl (1997) presented high-speed polarimetry obtained at the AAT in June 1993, which supports this view. In the AAT data the circular polarization is negative between $\phi=0.53-1.10$, i.e. for most of the bright phase, and becomes positive between phases 0.10 and 0.27 . Sohl interpreted this observation as evidence for a second accretion region with different field polarity. This interpretation seems unlikely to us given the smooth and continuous variation of the wavelength of the cyclotron humps indicating that they originate mainly from one region and due to the lack of a clear signal from a second region in the eclipse light curves (see below). We interpret the sign reversal of the circular polarization and the reversal in the wavelength shift of the cyclotron humps as due to the local orientation of the field line(s) in the cyclotron emitting region.

In our calculations we use cyclotron models as described and used earlier by us (Schwope et al. 1990). We note, that we are modeling here mainly the optically thin part of the cyclotron spectrum, where the humps are formed. Optically thick radiation is also present and may originate from somewhat different locations. For this reason our light curve model is incomplete and somewhat uncertain. In principle, a large number of free parameters determine the phase-dependent cyclotron spectrum like the field orientation, the field strength, the plasma temperature, the specific mass accretion rate, the extent of the region, and the white dwarf mass. With this large number of free parameters it is almost impossible to achieve a unique fit to the data, and we restrict ourselves to a onedimensional homogeneous emission region with fixed temperature at a reasonable location.
By a careful analysis of the PSPC X-ray light curve we have determined the location of the X-ray emitting accretion spot (Schwope et al. 2001) and the likely orientation of the magnetic axis. We refer to this as "standard geometry", it is characterized by its longitude $\chi_{\mu}=45^{\circ}$ and its co-latitude $\delta_{\mu}=15^{\circ}$. In the present analysis we choose our geometry in accord with this standard geometry but allow for a displacement of the cyclotron emitting spot from the X-ray emitting spot along a line defined by the footpoints of dipolar magnetic field lines connecting the ballistic accretion stream and the white dwarf, i.e. the footline of the accretion curtain found in Schwope et al. (2001).

The dipolar field model in the standard accretion geometry predicts that the local field vector is inclined away from the observer at the beginning of the bright phase and towards the observer at the end (see Fig. 6, a full explanation of that figure is given in Sect. 4.2), hence it cannot explain the observed reversal of the cyclotron humps. The observations require an opposite orientation of the field line in the accretion spot. Our modeling shows that the extra longitudinal tilt with respect to the white dwarf surface is of the order of $15^{\circ}$.

In our modeling we also allow for a vertical displacement of the emission region in order to reflect the length of the optical bright phase. After some tests and visual screening of the results we achieved a fit reflecting the main observational features. The accepted model shown in Figs. 3 and 4 is characterized by the following parameters: plasma temperature $k T=7 \mathrm{keV}$, magnetic field strength $B=34.1 \mathrm{MG}$, location of the spot defined by its co-latitude $\delta_{\mathrm{s}}=23^{\circ}$ and its azimuth $\chi_{\mathrm{s}}=29^{\circ}$, local field in the spot defined by its co-latitude $\delta_{\mathrm{f}}=27^{\circ}$ and its azimuth $\chi_{\mathrm{f}}=44^{\circ}$, and a height of the region above the stellar surface of $h=0.03 R_{\mathrm{wd}}\left(=2.4 \times 10^{7} \mathrm{~cm}\right)$. The location of the humps is a sensitive function of magnetic field strength, once the plasma temperature is fixed; the uncertainty then is of the order of $0.2 \mathrm{MG}$. The systematic uncertainty due to the poorly constrained temperature of the cyclotron spot is of the order of 0.5 MG. The (cyclotron) spot location corresponds to the very "near" end of the accretion arc, which connects directly to $L_{1}$, i.e. apart by some $12^{\circ}$ from the soft X-ray accretion spot. The value of $\delta_{\mathrm{f}}$ was chosen in accordance with a dipolar geometry, whereas the value of $\chi_{\mathrm{f}}$ was adjusted under the requirement to reach below the polarization horizon at the end of the bright phase. The size parameter $\log \Lambda=3.5$ was adjusted at a low value so that the cyclotron radiation in the two harmonics in concern here is completely optically thin. The height was chosen in order (a) to get an as extended bright phase as possible, (b) to match the start of the bright phase and (c) not to view from below the polarization horizon at the start of the bright phase.

The model reflects the main observed features, the monotonic motion of the harmonics during the first part of the bright phase, the reversal of the motion at $\phi=1.15$, the doublehumped shape of the light curve and an optical bright phase extending up to phase $\phi=1.32$ (in accordance with polarization observations obtained two months earlier). The fit is not completely successful in reflecting a bright phase lasting until phase 0.4 (although the exact length of the bright phase is difficult to determine observationally), and in the asymmetry of the redshift of the cyclotron lines in the first and the second hump. 




Fig. 3. Phase-resolved cyclotron model spectrum compared with the observed wavelengths of cyclotron harmonic peaks of HU Aqr. The parameters of the model are explained in the text.

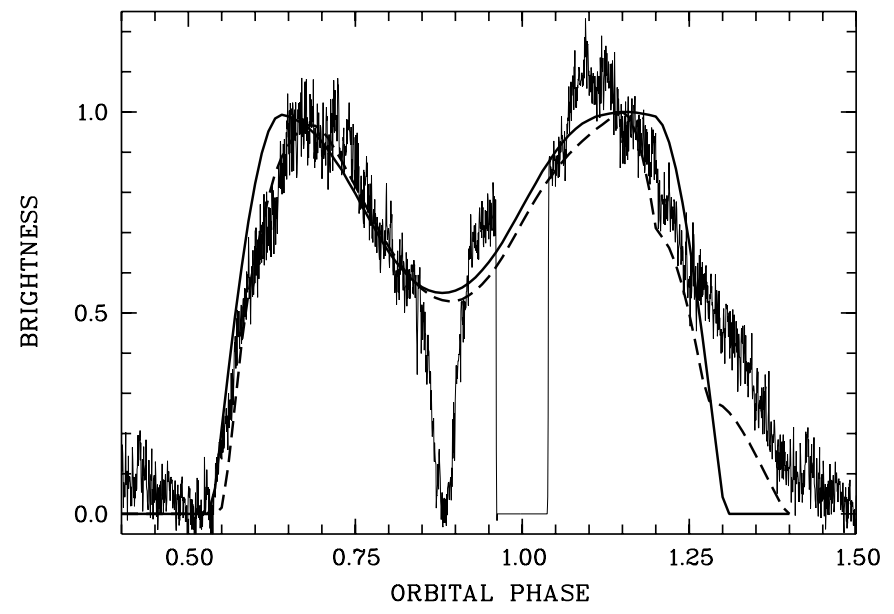

Fig. 4. Cyclotron fits to the $R$-band light curve of HU Aqr (same as in Fig. 2). The solid line is the result of a point source cyclotron model with same parameters as in Fig. 3. The dashed line is the result of a coarse arc model with same parameters as in Fig. 8.

However, in any case the model requires an orientation of the local field vector which is not in accordance with the simple centred dipole approximation. The cyclotron emitting spot as described here is displaced by $\sim 12^{\circ}$ from the $\mathrm{X}$-ray spot (indicated by $\mathrm{a}+$ in Fig. 6 ). This is a surprisingly large offset and implies the existence of hot plasma without significant X-ray emission. Further evidence for such an offset is derived from an analysis of the optical/X-ray light curves at eclipse ingress and egress, as presented in the next section.

\subsection{The high-state accretion arc in HU Aqr}

In Fig. 5 we show optical (MCCP $B$ and $R$ ) and X-ray (ROSAT PSPC) light curves with high time resolution.

Firstly we note, that the eclipse egress lasts much longer at optical wavelengths, both in $R$ and $B$, than at X-ray wavelengths. The same probably applies to the ingress, however, in the X-ray band the ingress is only barely resolved due to severe soft X-ray absorption in the accretion curtain. At optical wavelengths, the egress lasts longer than the ingress, $\sim 9 \mathrm{~s}$ and $\sim 4 \mathrm{~s}$, respectively. The slope of the light curve at ingress and egress is somewhat steeper in $R$ than in $B$, likely due to the non-negligible contribution of the white dwarf photosphere to the $B$-band. The much longer ingress/egress phases at optical wavelengths imply that the cyclotron emission region is much more azimuthally extended than the soft X-ray emission region. The short X-ray ingress/egress coincides with the end of the optical ingress/egress phase. This clearly shows that an accretion arc is the likely source of the optical emission with the soft X-ray emitting hot spot located at one end of the arc. As seen from the secondary star, this is the far end.

An accretion arc is naturally given as the footline of the accretion curtain. We test, whether the ensemble of foot-points of field lines of a centred dipole with a given orientation are sufficient to reflect the contact points of the observed light curves. In accordance with our standard accretion geometry as derived in Schwope et al. (2001) we assume a centred dipole with a co-latitude of the magnetic axis $\delta_{\mu}=16^{\circ}$ and an azimuth of $\chi_{\mu}=45^{\circ}$. As seen from the white dwarf, the ballistic accretion stream extends from the inner Lagrangian point $L_{1}$ at zero azimuth to an azimuth of about $50^{\circ}$ in the orbital plane. Using a stepsize of $\Delta \chi=1^{\circ}$ we compute the footpoints (position on the white dwarf surface defined by co-latitude and longitude/azimuth) of field lines which connect to the ballistic stream. The result is shown in Fig. 6. In addition, we compute for later use the orientation of the magnetic field vector in each foot-point. This vector is also characterized by two angles, a co-latitude and a longitude, and for each foot-point also plotted in Fig. 6 (lower ensemble of data points). The arc computed in this way stretches from longitude $29^{\circ}$ to $48^{\circ}$ and from about $23^{\circ}$ to $31^{\circ}$ in co-latitude. As mentioned in the previous section, according to this model the azimuth of the field vector is not inclined away from the observer at the end of the bright phase contrary to the observed circular polarization sign reversal and the motion of the cyclotron humps.

We compute the visibility function of the accretion arc (summed visibility of the foot-points) for an orbital inclination of $85.6^{\circ}$, a mass ratio $Q=M_{1} / M_{2}=4$ and a white dwarf mass $M_{1}=0.676 M_{\odot}$. This is shown as uppermost curve in Fig. 5. This model calculation predicts contact phases at ingress at -0.03862 and -0.03822 , i.e. an ingress interval of $3 \mathrm{~s}$, and contact phases at egress at 0.03952 and 0.04005 , i.e. an egress interval of $4 \mathrm{~s}$. While the former is in agreement with the observations, the latter interval is shorter than the observed one. In the optical, eclipse egress starts at about phase 0.039 . The observations, i.e. the early start of the eclipse egress, show, that the real arc is significantly more extended than compatible with our standard geometry. This is demonstrated in Fig. 7 where the eclipse geometry is shown for phases -0.03887 and 0.03887 , the contact phases where the optical eclipse ingress and egress start. The bundle of field lines shown there connects the ballistic stream with the white dwarf surface between the $L_{1}$ and the coupling region. The sketch shows, that the real arc must be larger in order to reproduce the observed contact phases of the 

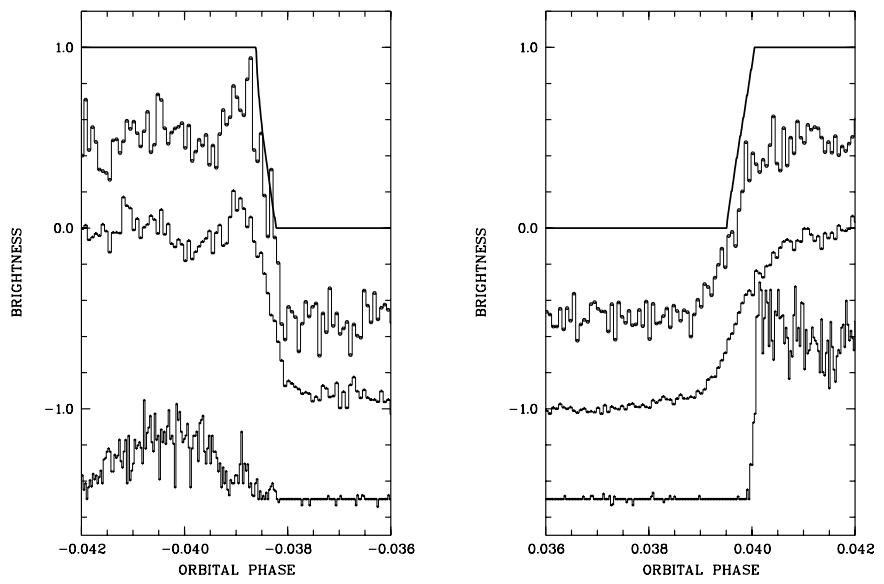

Fig. 5. Optical $R$ - and $B$-band and ROSAT PSPC X-ray eclipse light curves of HU Aqr. The optical light curves were normalized at ingress and egress to a stepsize of 1, the X-ray light curve was normalized to egress only. All curves are plotted with an offset along the ordinate of 0.5 units with respect to each other. The time interval along the abscissa corresponds to $45 \mathrm{~s}$ in both panels. The stream emission, which is significant during the first two minutes of the optical eclipses was approximated as low-order polynomial and subtracted before normalization. The top curve is the visibility curve of surface elements along the accretion arc, indicated as standard geometry in Fig. 6.

eclipse. The likely extent of the accretion region arc is down to azimuth $0^{\circ}$ and up to a co-latitude of $\sim 18^{\circ}$. The full accretion arc thus has a full opening angle of about $20^{\circ}$ corresponding to a linear dimension of $\sim 0.35 R_{\mathrm{wd}}$ (white dwarf radii).

\subsection{Cyclotron emission from the accretion arc}

A cyclotron model for the accretion arc has an almost "infinite" number of free parameters. Even if one approximates the arc as an ensemble of point-like emission regions with each one characteristic temperature, the number of variables is too large in order to achieve a unique solution. The mass accretion rate along the arc will unevitably be variable. As a consequence the plasma temperatures will vary along the accretion arc. Due to the different cooling behaviour as a function of the temperature the height of the emission will vary. This in turn changes the field strength in the emission region due to the strong radial dependence of the magnetic field.

Further complications arise from the fact, that the field geometry is more complex than a centered dipole. Hence we don't know the orientation of the field and the field strength at a given locus on the white dwarf's surface. All these parameter determine the shape of the cyclotron spectrum of a given elementary emission region.

A crude model spectrum can be created by just accounting for the end points and the center of the arc. We do so by assuming that the far end at $\delta_{\mathrm{s}}=31^{\circ}, \chi_{\mathrm{s}}=46^{\circ}$ has the highest temperature, since most of the matter is accreted there, and the near end at $\delta_{\mathrm{s}}=18^{\circ}, \chi_{\mathrm{s}}=0^{\circ}$ has the lowest temperature. We assume temperatures of $10 \mathrm{keV}$ and $3 \mathrm{keV}$, respectively, and use the same parameters for the central region at $\left(\delta_{\mathrm{s}}=23^{\circ}, \chi_{\mathrm{s}}=29^{\circ}\right)$ as in Sect. 4.1 above, i.e. $7 \mathrm{keV}$. A constant height of $0.015 R_{\mathrm{wd}}$ is assumed throughout. The lower height compared with the

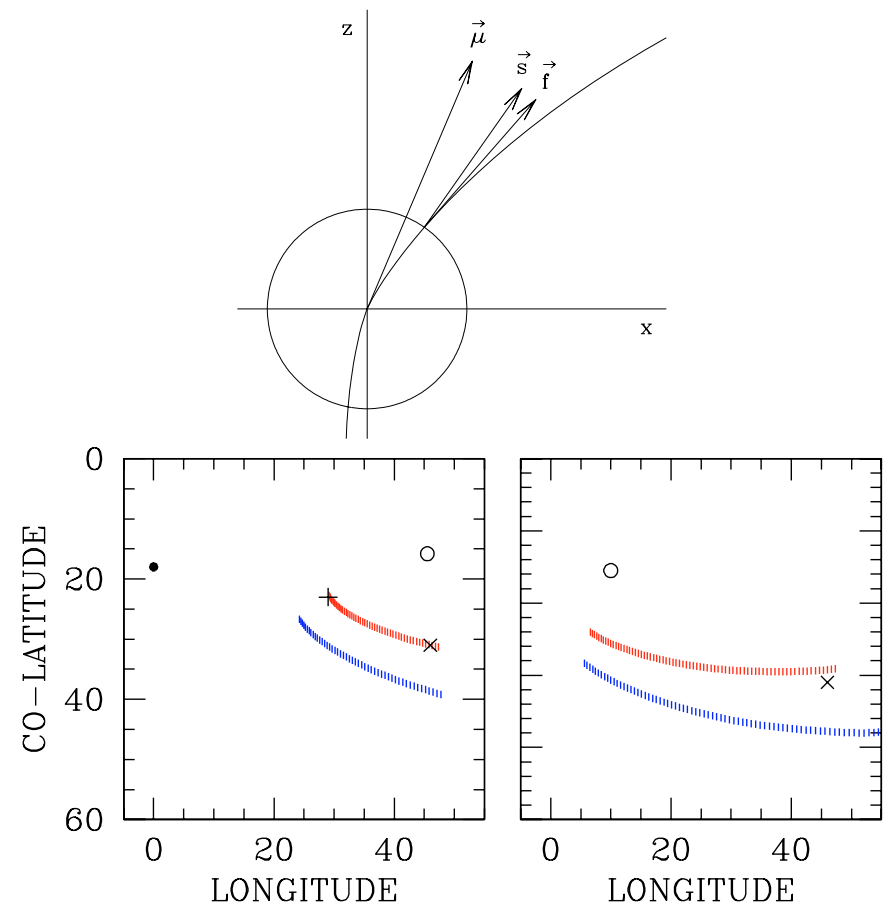

Fig. 6. (Top) Sketch of the dipolar geometry. The vectors $\boldsymbol{\mu}, \boldsymbol{s}, \boldsymbol{f}$ indicate the orientation of the dipolar axis, the location of an accretion spot at the foot-point of a dipolar field line and the orientation of the field line in the spot. (bottom) Accretion arcs in HU Aqr for two different orientations of the magnetic dipole axis (left - standard geometry, right - alternative geometry). The locus of the magnetic pole is indicated by the circles. The arcs are given as foot-points of dipolar field lines connecting the white dwarf and the ballistic stream. In each panel two different quantities are plotted, longitude and co-latitude of points on the white surface along the arc (coordinates of $s$ in the sketch above, upper row of data points in both panels), and longitude and latitude of the field line in the points (coordinates of vector $f$ in the sketch). These are computed for a centered dipolar configuration. The footline itself determines the visibility of the arc (plus the vertical extent), the orientation of the magnetic field determines the polarimetric and beaming properties. The left end of each arc connects to the $L_{1}$-point, the right end connects to the end of the ballistic stream in the orbital plane. The position of the soft X-ray emitting spot is indicated by the symbol $\times$. The position of the cyclotron emitting spot described in Sect. 4.1 is indicated by the symbol +. The third point of the simple arc model Sect. 4.3 is represented by a filled circle.

one-spot model (Fig. 3) reduces the visibility of the individual element and hence shortens the bright phase. This effect is completely balanced by the longitudinal extent of the arc (see Fig. 4).

For all three locations we further assume that the local field line is inclined by $10^{\circ}$ in azimuthal direction towards the observer at the start of the bright phase. The field strength assumed by us is 34.0, 34.1, and 34.4 MG at the near end, center and far end of the arc, respectively, and intensity weights of 4,2 , and 1 were given to the individual locations. The resulting model spectrum and light curve are shown in Figs. 8 and 4 (dashed line). The length of the bright phase is much better reflected by this rough arc model with less height of the emission region than by the one-spot model of Sect. 4.1. The motion of the cyclotron harmonics is similar to the one-spot model, 

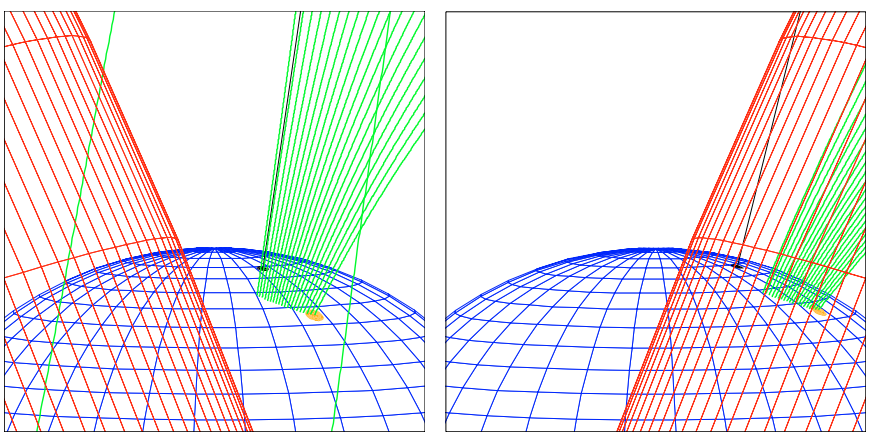

Fig. 7. Eclipse geometry of HU Aqr at phases -0.03887 and 0.03887 , i.e. at start of the optical eclipse ingress and egress. Shown are the loci of the accretion spot, the magnetic axis, magnetic field lines connecting to the ballistic stream and the shapes of the secondary's Roche lobe and of the white dwarf. The grid on the white dwarf has steps of $5^{\circ}$ in latitude and $10^{\circ}$ in azimuth. The X-ray emitting spot at the lower right end of the accretion arc is occulted lastly and re-appears lastly.

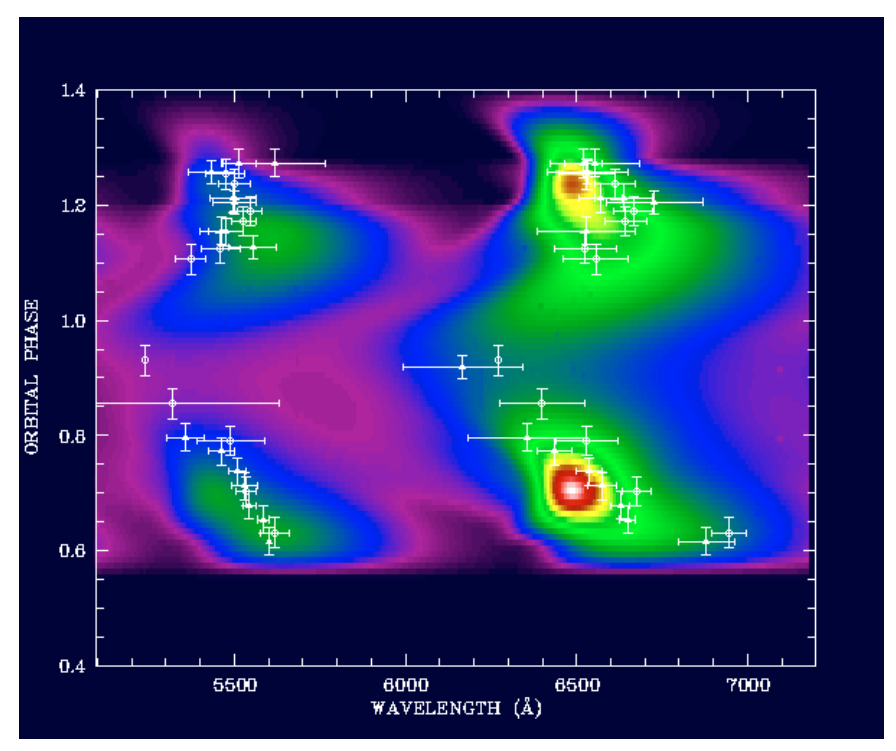

Fig. 8. Phase-resolved cyclotron model spectrum compared with the observed wavelengths of cyclotron harmonic peaks of HU Aqr. The parameters of the model are explained in the text.

a combined effect of the variable field strength in the three spots, the variable temperature, and the assumed orientation of the field vectors in the spots. In order to retain the asymmetric motion of the cyclotron humps we had to keep the tilt of the field vectors by $10^{\circ}$ in forward direction. Hence, the details of the eclipse light curves, the length of the optical bright phase and the motion of the cyclotron humps suggest the presence of an extended accretion arc of size $\sim 20^{\circ}$. The likely field orientation along the arc and the mere presence of an arc down to longitude zero are not compatible with the common assumption that the accreted matter couples via dipolar field lines to the ballistic stream. Even if we allow for immediate coupling at the $L_{1}$, we cannot reach longitude zero in our standard geometry (which otherwise gives excellent fits to the X-ray light curves and to Doppler tomograms). All these results suggest, that the near-field probably deviates from the centered dipole geometry.

There is one possible cure for these problems by chosing a completely different orientation of the magnetic axis, $\chi \simeq 0^{\circ}$, i.e. tilted towards the secondary star. Such a geometry cures two problems but creates a big new one. In such a geometry the matter leaving the $L_{1}$ has to run much further around the white dwarf until it couples onto field lines (again assuming a dipolar field geometry). Coupling occurs at an azimuth of about $70^{\circ}$, i.e. much later than suggested by the X-ray absorption dip. The matter then runs back towards the line connecting both stars. The corresponding accretion arc is more extended compared to our standard geometry and the field lines have a slight tilt towards the observer at start of the bright phase (the accretion arc for this alternative accretion geometry is also shown in Fig. 6). Hence, such a geometry has some advantage but the required late coupling is a serious problem. The soft $\mathrm{X}$-ray and optical absorption dips clearly show, that the bulk of matter couple to field lines at an azimuth of about $45^{\circ}$ instead of the required $70^{\circ}$. This inconsistency prevents us from accepting this alternative geometry as the final solution.

\section{Summary and conclusion}

We have analysed high-speed optical photometry and separated the cyclotron contribution to the $R$-band. This is essentially the fifth harmonic of a $\sim 7 \mathrm{keV}$ plasma in a field of $B \simeq 34 \mathrm{MG}$. The field strength derived here is somewhat lower than in our discovery paper (Schwope et al. 1993), combined effect of our ignorance of the exact geometry at that time which results in the choice of the wrong inclination angle of the local field and the higher assumed temperature in the original analysis.

Simultaneous low-resolution spectral observations showed pronounced cyclotron harmonic humps. The wavelengths of these humps could be traced throughout the optical bright phase between $\phi=0.6-1.3$. This and a detailed study of the eclipse light curves at optical and soft X-ray wavelengths clearly shows that the optical (cyclotron) emission region is much more extended than the soft X-ray emitting hot spot. Eclipse light curve modelling shows an accretion arc as the likely source of the cyclotron component. The arc seems to be more extended than compatible with accretion in our standard geometry. In this geometry an arc is formed at the footline of an accretion curtain which is raised along dipolar field lines between the ballistic stream and the white dwarf. The soft $\mathrm{X}$-ray emitting hot spot is located at the far end of the arc (as seen from the secondary star). The observations require a tilt of the field lines towards the observer at the start of the bright phase by about $10^{\circ}-15^{\circ}$ which again is hardly compatible with the standard accretion geometry. A change of the geometry by chosing a different orientation of the magnetic axis partially cures these problems but predicts a completely different coupling region than observed.

When fitted with a one-spot geometry, the cyclotron emission region is vertically more extended than the soft X-ray emission region with a height $h_{\mathrm{cyc}}=0.025 R_{\mathrm{wd}}$, compared to $h_{\mathrm{SX}}=0.015 R_{\mathrm{wd}}$. Recently, Fischer \& Beuermann (2001) solved the 1D radiation hydrodynamics of accretion regions 
in polars including the full angle-dependent radiative transfer in the cyclotron component. They derive the geometrical emission height, where most of the cyclotron radiation originates, to be $\sim 2 \times 10^{8} \mathrm{~cm}$ in the low $\dot{m}$-regime (specific mass accretion rate, per unit area). Our derived low value of the depth parameter $\Lambda$ suggests that we are indeed in the low $\dot{m}$ regime with $\dot{m} \simeq 10^{-3} \mathrm{~g} \mathrm{~cm}^{-2} \mathrm{~s}^{-1}$. The shock height by Fischer \& Beuermann is about an order of magnitude larger than observed in HU Aqr and suggests that energy loss through the side surfaces, not included in the Fischer \& Beuermann model, is responsible for the smaller observed emission height. The requirements posed to the height of the emission region are further relaxed, if the longitudinal extent of the region is taken into account. The height might then be compatible with the height of the soft X-ray emission region, i.e. $h_{\mathrm{cyc}} \simeq h_{\mathrm{SX}}=0.015 R_{\mathrm{wd}}$.

The integrated cyclotron flux, extrapolated into the ultraviolet and infra-red spectral ranges, is of the order of $3 \times$ $10^{11} \mathrm{erg} \mathrm{cm}^{-2} \mathrm{~s}^{-1}$. This is in excess of the bremsstrahlung flux in the ROSAT-PSPC observation of Oct./Nov. 1993, $F_{\mathrm{br}} \simeq 5.4 \times 10^{-12} \mathrm{erg} \mathrm{cm}^{-2} \mathrm{~s}^{-1}$, but clearly much lower than the integrated flux in the soft X-ray component, $F_{\mathrm{bb}} \simeq$ $4.4 \times 10^{-9} \mathrm{erg} \mathrm{cm}^{-2} \mathrm{~s}^{-1}$ (Schwope et al. 2001). The implied cyclotron luminosity and mass accretion rate are $L_{\text {cyc }} \simeq$ $\pi d^{2} F_{\text {cyc }}=3 \times 10^{31} d_{180}^{2} \mathrm{erg} \mathrm{s}^{-1}\left(d_{180}^{2}\right.$ : distance in units of $180 \mathrm{pc}$ ) and $\dot{M}=L R_{\mathrm{wd}} / G M=1.8 \times 10^{14} \mathrm{~g} / \mathrm{s}=3 \times 10^{-12} M_{\odot} / \mathrm{yr}$. Interestingly, the mass accretion rate estimated from the cyclotron component is about $50 \%$ of the mass accretion rate in the accretion curtain, which was estimated by Schwope et al. (2001) to be $\sim 6 \times 10^{-12} M_{\odot} / y r$. No significant soft Xray emission was detected from the accretion arc. These results suggest a coexistence of accretion regions with widely different specific mass accretion rates $\dot{m}$, a high $\dot{m}$-regime in the soft Xray emitting hot spot, and an accretion arc with moderate to low $\dot{m}$. Where the bulk of hard X-rays originate can hopefully be resolved by upcoming XMM-Newton observations and help to finally solve the famous soft X-ray puzzle of the AM Herculis stars.
Acknowledgements. We kindly acknowledge helpful comments of our referee, Dr. S. Potter. This project was supported in part by the Bundesministerium für Bildung und Forschung through the Deutsches Zentrum für Luft- und Raumfahrt e.V. (DLR) under grant number 50 OR 97068 and in part by the Deutsche Forschungsgemeinschaft (DFG) under grant Schw536/4-1.

\section{References}

Barwig, H., Schoembs, R., \& Buckenmayer, C. 1987, A\&A, 175, 327 Cropper, M., Mason, K. O., \& Allington-Smith, J. R. 1989, MNRAS, 236, 29

Fischer, A., \& Beuermann, K. 2001, A\&A, 373, 211

Glenn, J., Howell, S. B., Schmidt, G. D., et al. 1994, ApJ, 424, 967

Hakala, P. J., Watson, M. G., Vilhu, O., et al. 1993, MNRAS, 263, 61

Hakala, P. J. 1995, A\&A, 296, 164

Harrop-Allin, M. K., Cropper, M., Hakala, P. J., Hellier, C., \& Ramseyer, T. 1999, MNRAS, 308, 807

Harrop-Allin, M. K., Potter, S. B., \& Cropper, M. 2001, MNRAS, 326, 788

Rousseau, T., Fischer, A., Beuermann, K., \& Woelk, U. A\&A, 310, 526

Schwope, A. D. 1990, Rev. Mod. Astron., 3, 44

Schwope, A. D., \& Beuermann, K. 1990, A\&A, 238, 173

Schwope, A. D., \& Beuermann, K. 1997, AN, 318, 111

Schwope, A. D., Beuermann, K., \& Thomas, H.-C. 1990, A\&A, 230, 120

Schwope, A. D., Beuermann, K., \& Thomas, H.-C. 1993, A\&A, 271, L25

Schwope, A. D., Mantel, K.-H., \& Horne, K. 1997, A\&A, 319, 894

Schwope, A. D., Schwarz, R., Sirk, M., \& Howell, S. B. 2001, A\&A, 375,419

Stockman, H. S. 1988, in Polarized Radiation of Circumstellar Origin, ed. G. V. Coyne, S.J., A. M. Magalhães, A. F. J. Moffat, et al. (Univ. of Arizona Press)

Sohl, K. B. 1997, Ph.D. Thesis, Univ. Leicester

Wickramasinghe, D. T., Cropper, M., Mason, K. O., \& Garlick, M. 1991, MNRAS, 250, 692

Vrielmann, S., \& Schwope, A. D. 2001, MNRAS, 322, 269 